\title{
Ordentliche Ärztekammersitzung
}

Mittwoch, 30. April 2003, Solothurn

Die Sitzung beginnt um 9.30 Uhr und endet um 17.00 Uhr. Die Mittagspause dauert 1 Stunde. Weitere Traktanden werden der Reihe ihres Eintreffens nach an die bereits bestehende Traktandenliste angehängt.

\section{Tagesordnung}

\section{Begrüssung, Mitteilungen}

Jahresbericht: Berichtsperiode 2002/2003

\section{Jahresrechnung 2002 der FMH}

\section{Budget 2004 der FMH}

Festsetzung der Mitgliederbeiträge 2004.

\section{Statutenrevisionen}

a) VSAO-Sektionen Genève und Neuchâtel Basisorganisation?

b) Einbindung der Mitglieder des VLSS in die kantonalen Gesellschaften;

c) Statutarische Verankerung einer Unbedenklichkeitserklärung für Abrechnungen ärztlicher Leistungen;

d) FMPP (Foederatio Medicorum Psychiatricorum et Psychotherapeuticorum): Aufnahme als mitspracheberechtigte Organisation in die Gremien der FMH.

\section{KVG}

a) Orientierung über den Stand der Beratungen;

b) Beschluss über ein allfälliges Referendum der FMH zur 2. Revision des KVG.

\section{Eidg. Volksinitiativen}

a) Initiative der SPS: Gesundheit muss bezahlbar bleiben;

b) Initiative «Gleiche Rechte für Behinderte».

\section{TARMED}

a) Einführung UV-/MV-/IV-Bereich;

b) Stand der Verhandlungen in den Kantonen;

c) Dignitätserhebung;

d) Delegierte Psychotherapie.

\section{PR-Konzept der FMH}

Projekt SAT: Zusammenfassung der Tarifdienste kantonale Ärztegesellschaften Fachgesellschaften - FMH

\section{Weiterbildung}

\section{Schaffung von Fähigkeits- und Fertigkeits-} ausweisen

a) Schaffung des Fähigkeitsausweises «Vertrauensarzt»;

b) Schaffung des Fertigkeitsausweises «Phytotherapie»;

c) Schaffung des Fertigkeitsausweises «Gastroskopie»;

d) Schaffung des Fertigkeitsausweises «Endoskopische retrograde CholangioPankreatographie ERCP».

\section{KWFB: Einsitznahme neuer Gesellschaften}

a) APPM (Akademie für Psychosomatische und Psychosoziale Medizin): Einsitznahme in die KWFB;

b) SMSH (Schweizerische Ärztegesellschaft für Hypnose): Einsitznahme in die KWFB.

\section{Revision der Weiterbildungsordnung}

\section{Umgang mit Tätern}

\section{Varia}




\section{Séance ordinaire de la Chambre médicale}

Mercredi 30 avril 2003 à Soleure

La séance commence à $9 \mathrm{~h} 30$ et se termine à $17 \mathrm{~h} 00$. La pause de midi dure 1 heure. Les autres points à l'ordre du jour seront ajoutés à l'ordre du jour selon leur ordre d'arrivée.

\section{Ordre du jour}

Accueil, communications

Rapport annuel:

période sous revue 2002-2003

Comptes annuels 2002 de la FMH

\section{Budget 2004 de la FMH}

Fixation des cotisations de membre 2004.

\section{Révisions des statuts}

a) Sections de l'ASMAC de Genève et de Neuchâtel: organisation de base?

b) Intégration des membres de l'AMPHS dans les sociétés cantonales de médecine;

c) Ancrage réglementaire d'une déclaration attestant la conformité de la facturation des prestations;

d) FMPP (Foederatio Medicorum Psychiatricorum et Psychotherapeuticorum): admission en tant qu'organisation avec droit de discussion au sein des organes de la FMH.

\section{LAMal}

a) Information sur l'état des débats;

b) Décision quant à un éventuel référendum de la FMH sur la deuxième révision de la LAMal.

\section{Initiatives populaires}

a) Initiative du PS «La santé à un prix abordable»;

b) Initiative «Droits égaux pour les personnes handicapées».

\section{TARMED}

a) Introduction dans le domaine $\mathrm{AA} / \mathrm{AM} / \mathrm{AI}$;

b) Etat des négociations dans les cantons;

c) Recensement de la valeur intrinsèque;

d) Psychothérapie déléguée.
Concept de relations publiques de la FMH

Projet Comité de pilotage TARMED (CPT): concentration des services tarifaires SCM-SDM-FMH

\section{Formation postgraduée}

1. Création d'AFC et de CAT

a) Création de l'attestation de formation complémentaire «médecin-conseil»;

b) Création du certificat d'aptitude technique «phytothérapie»;

c) Création du certificat d'aptitude technique «gastroscopie»;

d) Création du certificat d'aptitude technique «cholangio-pancréatographie endoscopique rétrograde» (ERPC).

\section{Droit de siéger à la CFPC pou:}

a) l'Académie pour la médecine psychosomatique et psychosociale (AMPP);

b) la Société médicale suisse d'hypnose (SMSH).

3. Révision de la Réglementation pour la formation postgraduée (RFP)

\section{Traitement des auteurs de délits}

\section{Divers}

